\title{
Echocardiographic diagnosis of large fungal verruca attached to mitral valve
}

\author{
Richard C. Pasternak, David S. Cannom, and Lawrence S. Cohen \\ From the Section of Cardiology, Department of Internal Medicine, Yale University School of Medicine, \\ New Haven, Connecticut, U.S.A.
}

In a patient with endocarditis due to Candida tropicalis echocardiograms from mitral valve vegetations were found to mimic the typical pattern of a left atrial myxoma. A mass was shown occupying the mitral orifice posterior to the anterior mitral leaflet; densities also appeared in the left atrium. Though these echocardiographic findings were consistent with the diagnosis of a left atrial myxoma, there were other distinctive differential diagnostic features. Other diagnostic possibilities must, therefore, be considered in the interpretation of echocardiograms which suggest left atrial tumour.

\section{Case report}

A 63-year-old man was transferred to Yale-New Haven Hospital from a chronic care institution because of dizziness and increasing weakness of two days' duration. He had been in good health until 10 months before this transfer, when he was admitted to another hospital elsewhere, having woken with weakness of his right arm and ataxia. A grade 3/6 apical holosystolic murmur radiating to the axilla was then heard. The cerebrospinal fluid was xanthochromic; a left carotid arteriogram was normal. He was discharged after 34 days $w^{1}$ th a diagnosis of cerebrovascular accident, of unknown aetiology. Blood cultures were negative at that time.

Three months before transfer he was admitted to the urology service of the other hospital for evaluation of a left upper quadrant abdominal mass. At operation a benign renal cyst was found and oversewn. Subsequently because of persistent fatigue he entered a chronic care institution and remained there for 48 hours before the transfer to Yale-New Haven Hospital.

On examination on admission to this hospital, he was an elderly, confused man. Oral temperature was $38.5^{\circ} \mathrm{C}$. He had bilateral Roth spots. There was a $3+$ left ventricular heave. A grade 3/6 holosystolic murmur was heard at the cardiac apex, with radiation to the axilla, but there was no diastolic murmur. There was bilateral facial paresis and a flaccid right hemiplegia. Laboratory data included a haematocrit of 31 per cent and a white blood count of 6400 cells $/ \mathrm{mm}^{3}$ with a shift to the left. CSF was again xanthochromic. Brain scan and skull $x$-ray films were normal. Chest $x$-ray film showed slight cardiomegaly, without evidence of left atrial enlargement, and clear lung fields. The electrocardiogram showed left anterior hemiblock and left atrial enlargement. Fig. 1 shows the echocardiogram obtained using standard techniques ${ }^{1}$ (Popp et al., 1969), with the transducer in the usual position for examination of the mitral valve. Towards the left of the figure abnormal densities appear posterior to the anterior mitral leaflet. Prolapse of the anterior or posterior leaflet is suggested throughout and is most clearly seen on the right hand side of Fig. 1. Fig. 2 is a scan from the aortic to the mitral region showing an irregular left atrial density during the latter half of diastole, disappearing shortly after the onset of the systole. Dense echoes are seen in the mitral orifice during diastole.

Blood cultures on admission grew Candida tropicalis. The patient was treated with amphotericin-B (50 mg daily); his condition remained stable until one week after admission when he had

${ }^{1}$ Unirad Series C Ultrasonoscope, Unirad Corp., Denver, Colorado. 


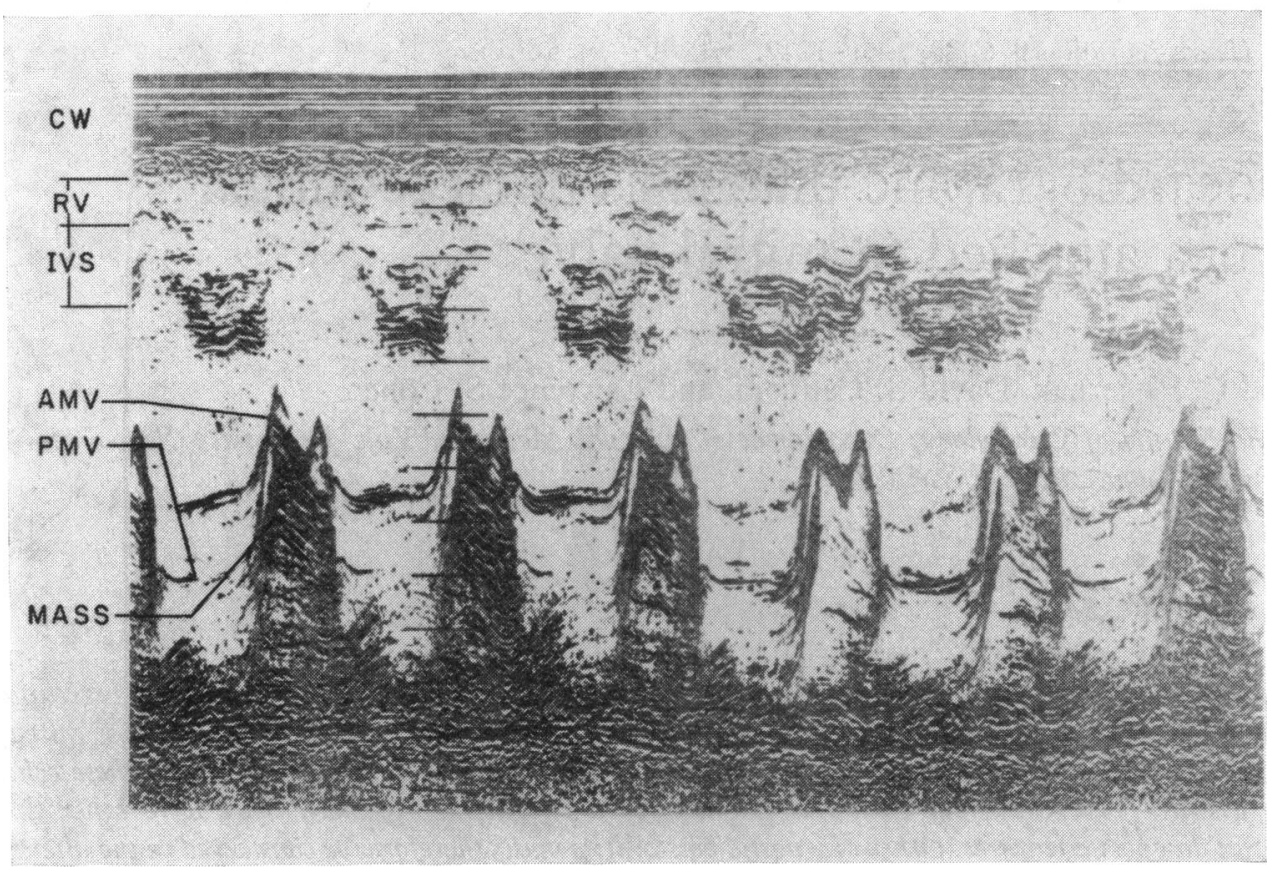

FI G. 1 Echocardiogram in normal mitral position. The normal anterior mitral leaflet (AMV) appears thin; the ' $a$ ' wave is prominent. The posterior mitral leaflet (PMV) is obscured by the mass during diastole. On the left side of the figure, dense echoes filling the mitral orifice during diastole appear, but are not seen during systole in this view. While these dense echoes did not disappear with reduction in gain, they became less prominent with slight transducer movement as seen in the right of the figure.

The exact origin of the structure labelled PMV in this illustration is controversial. Though we have regarded this as posterior mitral leaflet, it is possible that a structure resembling this might in fact represent a severely prolapsing leaflet or both leaflets together (Popp et al., 1974).

$A M V$-anterior mitral valve leaflet; $C W$-chest wall; IVS-interventricular septum; $P M V$-posterior mitral valve leaflet; $R V$-right ventricle.

further emboli with impaired consciousness, left hemiparesis, and absent pulses in the left leg. A left femoral embolectomy was performed and microscopical examination of the embolus showed thrombus and fungal forms.

On the following day, at cardiac catheterization, an angiocardiogram following injection into the pulmonary artery showed a spherical filling defect behind the mitral valve, moving with the posterior leaflet; there was significant prolapse of the posterior leaflet into the atrium during systole and the leaflet appeared thickened and nodular.

At operation next day, a large fungal mass was found to be attached to the posterior mitral valve leaflet. The mitral valve was excised and a StarrEdwards prosthesis was implanted. The operative specimen consisted of calcified and fibrous valve tissue with large, irregular, fibrinous vegetations easurinmg $2 \mathrm{~cm}$ at the greatest diameter. Histologically, masses of fungal organisms contained in a thrombotic mesh were seen. No myxomatous tissue was present.

Fever persisted and, after a protracted course complicated by respiratory and renal failure, he died on the 20th postoperative day.

\section{Discussion}

The occurrence of fungal endocarditis with the development of large fungal vegetations attached to the mitral or aortic valve is becoming increasingly common (Drutz, 1971). Once candida was grown from the blood cultures there was little doubt, on clinical grounds, that our patient had fungal endocarditis. The echocardiogram was, however, obtained before cultures became positive and 


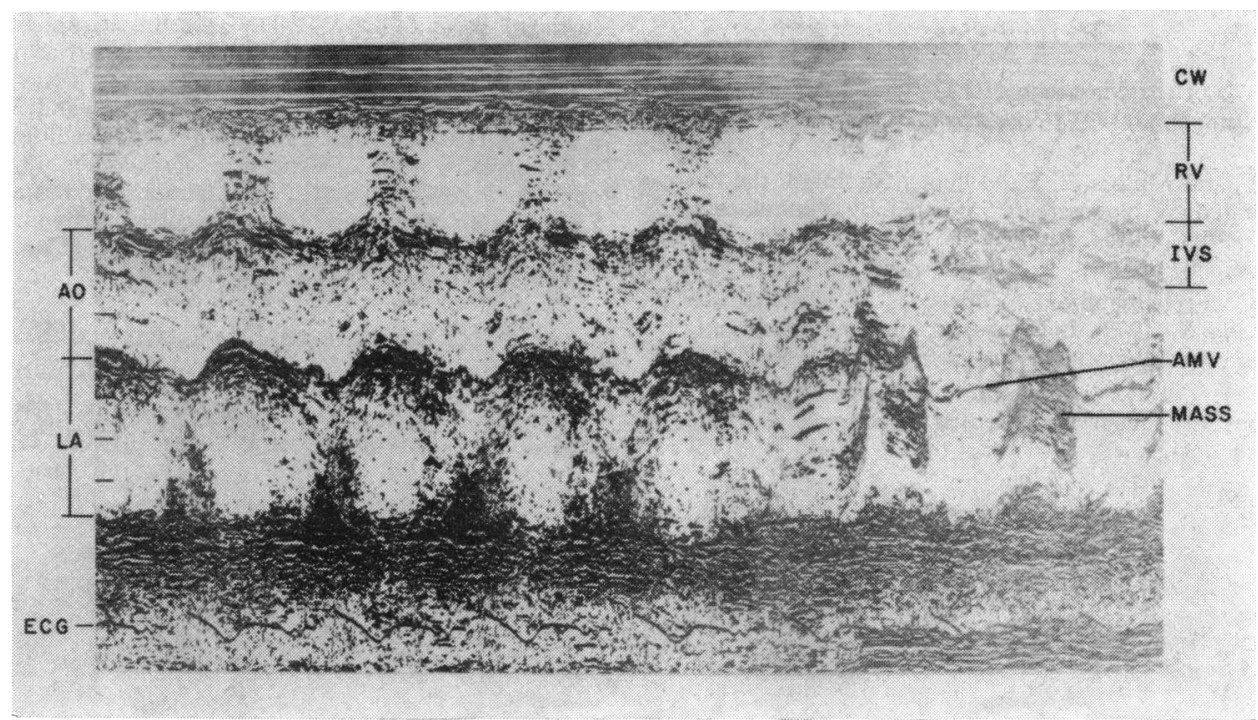

FIG. 2 Echocardiographic scan of aortic and mitral regions. The mass is posterior to the anterior leaflet $(A M V)$ during diastole. Echocardiographic densities are shown in the left atrium ( $L A)$ during late diastole and early systole. AMV-anterior mitral valve leaflet; $A O$-aortic root; CW-chest wall; ECG-electrocardiogram; IVS-interventricular septum; LAleft atrium; $R V-$ Right ventricle.

the question of left atrial myxoma was raised. It is understandable that a fungal verruca on the mitral valve should mimic a left atrial myxoma echocardiographically, in view of their similar size and site. When positive blood cultures were obtained the possibility of an infected myxoma was considered. Though rare, such cases have been reported (Dick and Mullin, 1956), with candida as the infecting organism.

Dillon and his colleagues have reported on the echocardiographic findings in 8 patients with bacterial endocarditis (Dillon et al., 1973). In all cases vegetations were between 2 and $8 \mathrm{~mm}$ in size, and thus were much smaller than the lesion reported in our patient $(2 \mathrm{~cm}$ at greatest diameter). The typical manifestations included apparent valvular thickening with a 'shaggy appearance'. The initial diagnostic uncertainty in this case resulted from the echocardiograms illustrated in Fig. 1 and 2. The authors of recent echocardiographic study of left atrial myxoma have observed that 'the recording of abnormal echoes from the region posterior to the anterior leaflet of the mitral valve during ventricular diastole is one of the most reliable manifestations of a tumour' (Martinez, Giles, and Burch, 1974). In addition, in a recent published report such a pattern is described as 'pathognomonic' of the condition (Case Records of the Massachusetts General Hospital, 1973). Dillon et al. (1973) have discussed the differentiating features between a myxoma and endocarditis. With tumour the posterior leaflet is not seen, the extra echoes appear to be unattached to the valve leaflet, and finally the tumour can frequently be seen as multiple irregular echoes in the left atrium. Though abnormal multiple echoes appear in the left atrium of our case, their timing (late diastole and early systole) is inconsistent with the usual myxoma pattern. Thus, this case shows that the demonstration of an abnormal mass posterior to the anterior mitral leaflet is insufficient evidence on which to make the diagnosis of left atrial myxoma.

Our experience with this case confirms that caution must be exercised in the interpretation of echocardiograms thought to show a left atrial myxoma. Other diagnoses, including bacterial and fungal endocarditis, must be considered. Echocardiography, however, is still a valuable screening investigation in patients with suspected lesions of the mitral valve. With the experience of cases such as the one reported here the range of conditions recognized by echocardiography widens further. Moreover, serial echocardiograms may be useful when such patients are treated medically; it may then be possible to observe repression of vegetations in endocarditis. 


\section{References}

Case Records of the Massachusetts General Hospital (1973). (Case Number 42-1793.) New England fournal of Medicine, 289, 853.

Dick, H. J., and Mullin, E. W. (1956). Myxoma of the heart complicated by bloodstream infection by staphylococcus aureus and Candida parapsilosis. New York fournal of Medicine, 56, 856.

Dillon, J. C., Feigenbaum, H., Konecke, L. L., Davis, R. H., and Chang, S. (1973). Echocardiographic manifestations of valvular vegetations. American Heart fournal, 86, 698.

Drutz, D. J. (1971). In: Medical Staff Conference, The spectrum of fungal endocarditis. California Medicine, 115 (Oct.), 34.
Martinez, E. C., Giles, T. D., and Burch, G. E. (1974). Echocardiographic diagnosis of a left atrial myxoma. American fournal of Cardiology, 33, 281.

Popp, R. L., Brown, O. R., Silverman, J. F., and Harrison, D. C. (1974). Echocardiographic abnormalities in the mitral valve prolapse syndrome. Circulation, 49, 428.

Popp, R. L., Wolfe, S. B., and Hirata, T., et al. (1969). Estimation of right and left ventricular size by ultrasound. A study of echoes from the left ventricular septum. American fournal of Cardiology, 24, 523.

Requests for reprints to Dr. Lawrence S. Cohen, Department of Internal Medicine, Yale University School of Medicine, 333 Cedar Street, Room 87 LMP, New Haven, Connecticut 06510, U.S.A. 